Volume 8, No.1.6, 2019

International Journal of Advanced Trends in Computer Science and Engineering

Available Online at http://www.warse.org/IJATCSE/static/pdf/file/ijatcse1981.62019.pdf

https://doi.org/10.30534/ịatcse/2019/1981.62019

\title{
A Grey Wolf Optimizer based Support Vector Machine for Classification of Finger Movement
}

\author{
Nurazrin Mohd Esa ${ }^{1}$, Azlan Mohd Zain ${ }^{2}$, Mahadi Bahari ${ }^{3}$ \\ ${ }^{1}$ Applied Industrial Analytics (ALIAS), School of Computing, Faculty of Engineering, Universiti Teknologi \\ Malaysia (UTM), Skudai, Malaysia \\ ${ }^{3,4}$ Information and Service Systems Innovation (ISSI), School of Computing, Universiti Teknologi Malaysia
}

(UTM), Johor, Malaysia

\begin{abstract}
Finger development acknowledgment framework is generally connected in numerous apply autonomy and assistive hand gadgets. The efficiency of the recognition system depends on their accuracy which highly related to their classification technique in this system. Among the classification, the most establish and outstanding method is Support Vector Machine (SVM). However, the efficiency of SVM can be optimizes by identification of optimizes of their parameter. The newest popular swarm intelligent is grey wolf optimizer (GWO). This GWO is popular because their efficiency in exploration and exploitations. In this study, we mainly concentrate on the parameter optimization of SVMs, which has gained great attentions in the past several years. This paper exhibits a creative arrangement framework for hand signals utilizing 2-channel surface electromyography investigation. The framework created utilizes the Support Vector Machine classifier, for which the piece capacity and parameter streamlining are directed moreover by the Grey Wolf Optimizer. The experimental result in this study is based on accuracy compared between the ten fingers movement based on SVM and proposed hybrid GWO-SVM and GWO-SVM result based on three different feature extraction methods. The results show that hybrid GWOSVM obtained better accuracy compared to the others.
\end{abstract}

Key words : Myoelectric control, Time Domain, Feature extraction, Classification, Support vector machine

\section{INTRODUCTION}

In rehabilitation, the research topic that always been an open question is the development of an advanced human-machine interfaces by using biomedical signal such as myoelectric signals. Preparing myoelectric motion in myoelectric control is new advancements that including a couple of steps, for example, detection, handling, order and use of myoelectric signs to control human-helping robots or recovery gadgets. Myoelectric control utilized the Myoelectric signals (MES) which contain rich data from which a client's expectation as a solid withdrawal to be distinguished. The number of channels to obtain MES data is important factors that affected the performance of myoelectric control system. Research by [1] has shown a clear trend; as the number of channels increases, the performance of the classifier increases. The increase is not linear; it quickly reaches a high value of approximately $90 \%$ as eight channels are added and asymptotically approaches a maximum of approximately $95 \%$ as the final eight channels are added.

Figure 1 shows the process of myoelectric control of hand prostheses fully in diagram. The process starts with the data acquisition follow by data segmentation, feature extraction and classification before the identified output sent to the digital controller. Then, the digital controller will command the prostheses to works based on identified output. The myoelectric process is starting from Data segmentation to the classification process.

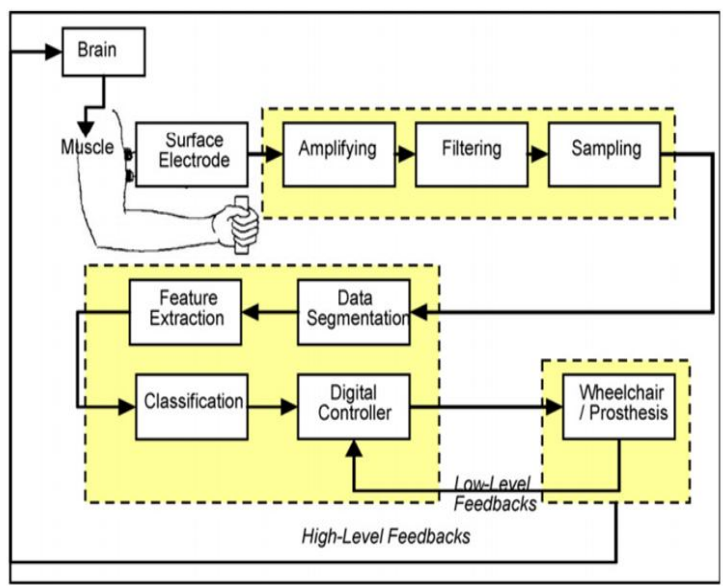

Figure 1: The process of Myoelectric Control of Hand prostheses[2]

Among these stages, the main contributor to system recognition performance is classification technique implemented. For classification, Support Vector Machine (SVM) had been proven widely in many fields of studies to be powerful classification tools [3-5]. [6] stated that the theory of SVM is based on structural risk management (RSM) idea. They linearly isolate two dataset by setting a hyper-plane between them that the distance of the two informational collections to hyper-plane is maximally vast. [7] stated that one of the aspects that offer priority to SVM over different classifiers is that it gives just a single solution. This implies SVM is a superior classifier than numerous layer perceptron (MLP) neural systems (NN), which gives 
you more than one solution relying on local minimum. Subsequently, we executed the OVA multiclass SVM to group the ten finger developments. Since the characterization exactness come about by SVM relies upon bit and punishment parameters, tuning these parameters assumes a key job in the execution of this procedure. Parameter $\mathrm{C}$, which controls the preparation mistake and speculation execution of SVM, and the part work parameters are the most critical parameters to be tuned. In this paper, since the RBF was utilized as the piece work, the $\sigma$ parameter of this capacity should be tuned. Albeit vast estimations of $\mathrm{C}$ cause high exactness rate in the preparation stage, the precision in the testing stage is low. Too little estimations of $\sigma$, then again add to inadmissible grouping rates. Since various estimations of parameter $\sigma$ has direct effect on the dividing of highlight space, deciding ideal incentive for this parameter is increasingly significant.

The most essential methodology in SVM characterization improvement is controlling the slackfactors punishment weight (C) and searching for the best exchange off between permitting misclassification blunders and summing up the model. High estimations of $\mathrm{C}$ will to a great extent punish misclassified models, and in this manner the subsequent hyperplane will be one that firmly keeps away from order mistakes, notwithstanding while giving up speculation. At last, a $\mathrm{C}=1$ will lead into a hard-edge SVM conduct. Then again, low qualities just delicately punish misclassifications, and the outcome may be an incorrect partition [8]. Lattice look strategy is another basic method to discover appropriate parameters for SVM. This technique requires a matrix look over the parameter space. The parameters shift with the fixed advance size through a wide scope of qualities. The execution of every parameter blend is surveyed by some execution measure. Network scan is appropriate for change of not very many parameters and does not perform well by and by in light of the fact that it is perplexing in calculation and tedious.

This paper handles the distinguished on ten finger movements utilizing surface EMG data in view of signal processing methods. The EMG signals were obtained utilizing two electrode patch on two particular muscles of the hand. In this work we analyzed three sorts of time domain include feature extraction strategies in view of classification accuracy of SVM techniques. Our outcomes demonstrate that the combination of RMS and Hudgins feature set demonstrate the best average classification accuracy for all ten fingers developments. The rests of this paper is organized as takes after. Segment II depicts the materials and methods for finger recognition system. The result and discussion are featured in Section III and the conclusion of this study show up in Section IV.

\section{MATERIAL AND METHODS}

This section introduces methods applied for EMG based classification of finger movement using SVM as classification method. The procedure begins with EMG flag procurement stage, pre-preparing of EMG flag, include extraction and in conclusion grouping staged. Figure 2 demonstrates the flowchart of this procedure. The procedure of myoelectric control of finger development beginning with the procurement of EMG flag pursued by preparing process which incorporate the information division and highlight extraction process. The data extracted then will be classified and desired output will be produce.

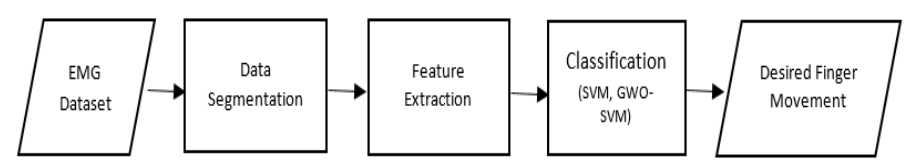

Figure 2: The process of Myoelectric Control of Hand Prostheses

\subsection{Acquisition of EMG signal}

EMG signal information procured from [9]. Allude from [9], the EMG data was accumulated using two EMG channels (Delsys DE 2.x arrangement EMG sensors). At that point, this flag gathered been prepared by the Bagnoli Desktop EMG Systems from Delsys Inc. Amid gathering information try, two channels of sensor immovably sticks into the skin by use of 2-space glue skin interface. A conductive cement reference cathode (Dermatrode Reference Electrode) was utilized on the wrist of each subject. Figure 4(a) and 4(b) demonstrates the pictures on the situation of the terminal fix on individual wrist. The EMG signals assembled from the cathode were amplified using a Delsys Bagnoli-8 amplifier to a total get of 1000. A 12-bit simple to computerized converter (National Instruments, BNC-2090) was used to test the banner at $4000 \mathrm{~Hz}$; the flag data was then acquired using Delsys EMGWorks Acquisition programming. The ten single and squeeze development that have been recognized are hand close (HC), list (I), little (L), center $(\mathrm{M})$, thumb $(\mathrm{T})$, ring finger $(\mathrm{R})$ and the squeeze development from blend of thumb and record (T-I), thumb and little (T-L), thumb and center (T-M) and thumb and ring fingers (T-R). Figure 5 demonstrate the ten kinds pictures of better development.

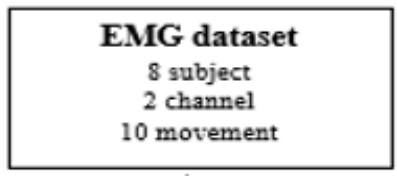

Figure 3: The information of EMG dataset

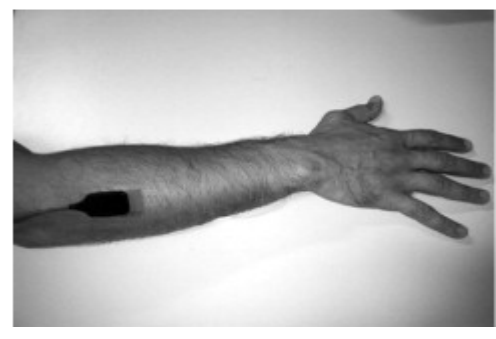

Figure 4(a): Position of sensor. [7]

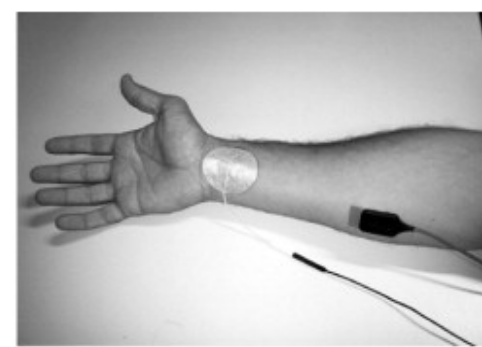

Figure 4(b): Position of sensor2 [7] 


\subsection{Data segmentation}

A segment or window is a sequence of data limited in a time slot, which is used to estimate signal features [2]. Data segmentation or windowing is a procedure of grouping of information restricted in a schedule opening, which is utilized to estimate signal features [10]. The natures of raw EMG signal contain large information in insignificant ways. However, this situation can change if this raw data been manipulated and convert into feature space data that contain high information. To convert it, the windowing process is compulsory [11]. The issues in windowing process are the determination of width for each segment of windows. If the width of segment is to short, it will lead to bias feature estimation while if too long cause likely to failed in realtime because high computational operations.

There are two common type of windowing which are disjoint and overlapping windows. In overlapped windowing, the length and increment are important parameter. Increment variable is represents the time interval between the neighbor segments. The guideline on choosing the time interval is the length needs to be larger than processing time but must be less than segment length. [9] stated that the overlapping windowing scheme produces better performance when comparing the classification accuracy, but if the data is too large it will lead to higher computational cost in the training and testing phase. The different between disjoint windowing is low accuracy with lower computational cost compared from disjoint which there are always had an overlapped segment and required more window to segment all the data. In this section, it is explained the results of research and at the same time is given the comprehensive discussion. Results can be presented in figures, graphs, tables and others that make the reader understand.

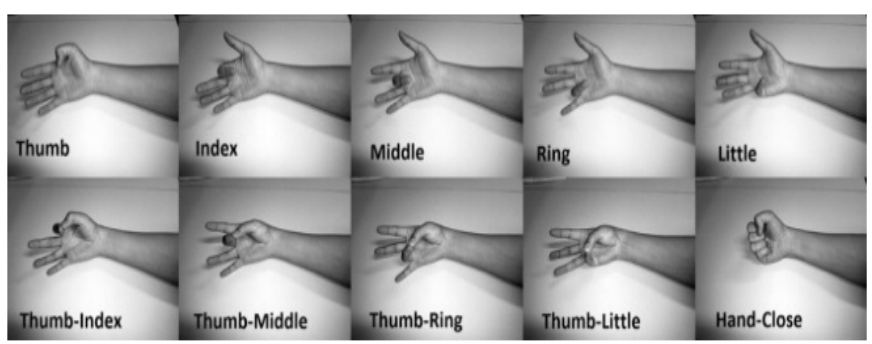

Figure 5: Types of finger movement studied. [18]

\subsection{Feature Extraction Phase}

Feeding the EMG signal as a raw time sequence, directly into the classifier causes degrading the classification performance. Raw EMG signal contains large sequence of input and varies in randomness that causes complexity. To caters the problem due to the curse dimensionality, feature extraction process consequently implements in order to condense only important EMG parts represent each classes ([15],[12]).The features were extracted using a time domain feature set which consists of mean absolute value (MAV), waveform length (WL), zero crossings (ZCs), slope sign changes (SSC) and root mean square (RMS). All features were extracted by using Matlab code provided by [12]. The description of each feature set uses is described briefly

\section{1) Mean Absolute Value (MA V)}

MAV is the average of the absolute value of the EMG signal amplitude in a sample of data. It is widely famous one as commonly used in EMG signal recognition (e.g. [13], [14]). However, there is the limitation of using MAV as single feature training set which is there are possibilities that different classes may contain the same mean value. But it is still can discriminated best depends on the variation of classes. The formula of extracting the MAV is as

$$
M A V=\frac{1}{N} \sum_{i=1}^{N}\left|x_{i}\right|
$$

\section{2) Zero Crossing (ZC):}

Zero crossing $(\mathrm{ZC})$ in time domain defines as the measure of frequency information of the EMG signal (e.g. [13],[15]). The value represents number of times that the amplitude of EMG signal passes the zero amplitude axes. The formula of extracting the $\mathrm{ZC}$ is as

$Z C=\sum_{i=1}^{N-1}\left[\operatorname{sgn}\left(x_{i} \times x_{i+1}\right) n\left|x_{i}-x_{i+1}\right| \geq\right.$ threshold $]$

3) Waveform Length (WL)

Waveform length (WL) in time domain features represents cumulative length of the EMG waveform over the time segment. It usually uses to identify the complexity of EMG signal sequence ([13], [10]). Wavelength (WAVE) is another representative name for this feature. The formula of extracting the WL is as

$$
W L=\sum_{i=1}^{N-1}\left|X_{i+1}-X_{i}\right|
$$

4) Slope Scope Change (SSC)

Similarly, to the ZC, Slope Sign Change (SSC), is a method represent frequency information of the EMG signal ([13], [15]). The different with $\mathrm{ZC}$ is the value represent cumulative numbers of times that slope of the dataset change sign either negative to positive value or conversely. It is performed using threshold function among three sequential segments changes for avoiding the background noise EMG signal. The mathematical formula of SSC is as

$S S C=\sum_{i=1}^{N-1}\left[f\left(x_{i} \times x_{i+1}\right) \cap\left|x_{i}-x_{i+1}\right| \gg\right.$ threshold $]$

\section{5) Root Mean Square (RMS)}

Another popular commonly used as features set is root mean square (RMS) ([17], [16]). The formulation of RMS is quite similar to standard deviation where amplitude 
modulated modeled related to non-fatiguing and constant force. The mathematical formula of RMS is as

$R M S=\sqrt{\frac{1}{N} \sum_{i=1}^{N} x_{i}^{2}}$

\subsection{Classification using GWO-SVM phase}

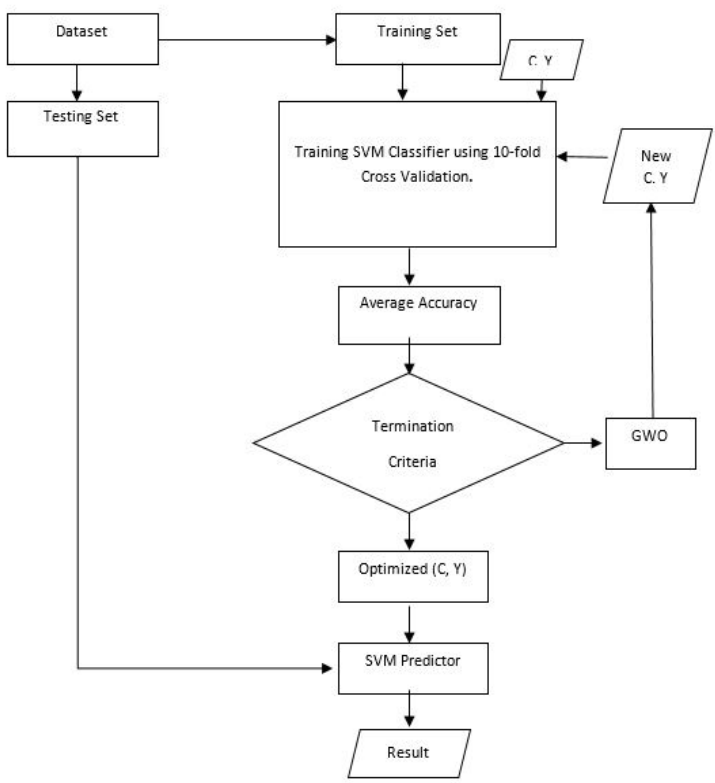

Figure 6: Framework of GWO-SVM algorithm

Higher accuracy, a smaller number of computational time and low storage space are the characteristic that measure the efficiency of recognition system. Nowadays, there are many classifiers that capable in providing the high efficiency to the system. Among them, SVM fulfill almost all the criteria of the effective classifier. Study by [18] shows that SVM is superior in terms of classification in recognizing multiple hand motion compared to another algorithm. Research conduct by [19] and [10] confirmed the effectiveness of EMG pattern recognition using SVM methods. Hence, SVM had proven to be powerful classification tools [20]. SVM is kernel based classification that the theory is from structural risk management (SRM) idea [20]. It algorithm will design the hyper-plane that linearly separate two data sets that maximally large the distance between dataset of two classes. The kernels of algorithm in SVM also provides the ability of classify data in non-linear separation without increasing calculation cost significantly.

Figure 6 shows the proposed hybrid of GWO with SVM to identify the parameter value of SVM. GWO identify the optimize value of cost $(\mathrm{C})$ and sigma parameter.

\section{RESULTS AND DISCUSSION}

EMG motion for this examination procured from [18] which comprise of ten classes of individual and joined fingers developments including: the flexion of every one of the individual fingers, i.e., Thumb, Index, Middle, Ring, Little and the squeezing of consolidated Thumb-Index, Thumb-Middle , Thumb-Ring, Thumb-Little, and finally the hand close. The information obtained has just been Bandpass filtered between $20-450 \mathrm{~Hz}$ with a step filter actualized to evacuate the $50 \mathrm{~Hz}$ line impedance.

This study focused on enhanced the classification of ten finger movement using EMG signal. The experimental result of this study represent in Table 1 which represent the accuracy of SVM and proposed GWO-SVM. Based on the result, there are significant different between the accuracy of SVM and enhance SVM for certain movement such as thumb index and little finger recognition. However. There are slightly degrade result for thumb-little accuracy. But others result is similar with no different of their accuracy. However, the average classification accuracy of proposed GWO-SVM improved compared to the SVM alone.

Table 1: Table result of classification accuracy between standard GWO and GWO-SVM

\begin{tabular}{lcc}
\hline Movement & $\begin{array}{c}\text { Classification } \\
\text { accuracy SVM }\end{array}$ & $\begin{array}{c}\text { Classification } \\
\text { accuracy GWO-SVM }\end{array}$ \\
\hline Hand close & $96.67 \%$ & $96.67 \%$ \\
\hline Little & $80 \%$ & $83.33 \%$ \\
\hline Middle & $96.67 \%$ & $96.67 \%$ \\
\hline Index & $93.33 \%$ & $93.33 \%$ \\
\hline Ring & $86.67 \%$ & $86.67 \%$ \\
\hline Thumb & $96.67 \%$ & $96.67 \%$ \\
\hline Thumb & $93.33 \%$ & $96.33 \%$ \\
Index & $90 \%$ & $83.33 \%$ \\
\hline Thumb little & $90 \%$ & $90 \%$ \\
\hline Thumb & & $96.67 \%$ \\
\hline Middle & $96.67 \%$ & \\
\hline
\end{tabular}

The figure 8 shows the result of classification accuracy based of feature extraction method. Based on the table below, the combination of Hudgins and RMS shows better result compared to Hudgins and RMS. The more data used to represent the class will make better accuracy. However, this will affect the computational time. 


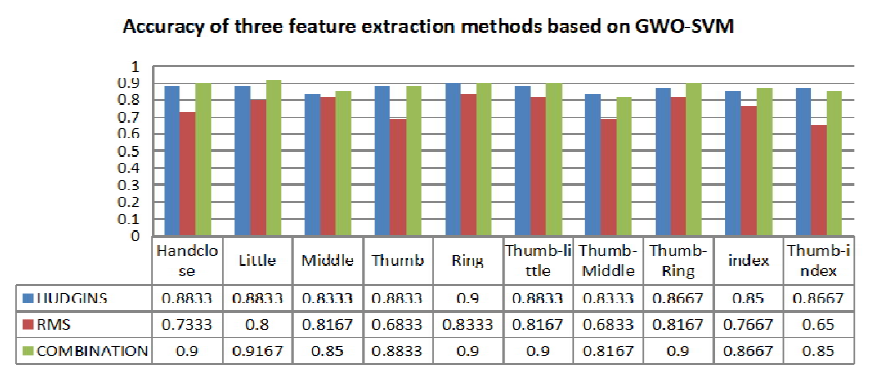

Figure 8: Result of classification accuracy between standard GWO and GWO-SVM

\section{CONCLUSION}

One versus all multiclass SVM architecture is successfully developed for identification of ten classes finger movement such as HC, L,M, I, R, T, T-I,T-L,T-M and T-R. OVA SVM is useful tool to classify the EMG signal with three group of feature set and ten class of EMG signal dataset. From three feature set which is single RMS, combination of RMS and Hudgins set and Hudgins set, all there feature give a competitive classification accuracy for all class, however based on average the combination of Hudgins and RMS features give betters compared to other two methods. Result of hybrid of GWO-SVM is better than SVM. However, the classification of little finger obtain the lowest accuracy because difficult to distinguish. Then, for the future work could use all these feature extraction technique with other classification methods such as Knearest neighbor (K-nn) and Neural Network or optimize the SVM by hybridization with optimization methods. Each has different advantage and its limitation. All these techniques can be explored further.

\section{ACKNOWLEDGEMENTS}

We would like to thank reviewer(s) for useful advices and comments. The authors greatly acknowledge the Research management Centre, (UTM) and Ministry of Higher Education Malaysia (MOHE) for financial support through the Fundamental Research Grant Scheme (FRGS) No. R.J130000.7828.4F868

\section{REFERENCES}

1. Kuiken, Todd A., Guanglin Li, Blair A. Lock, Robert D. Lipschutz, Laura A. Miller, Kathy A. Stubblefield, and Kevin B. Englehart. "Targeted muscle reinnervation for real-time myoelectric control of multifunction artificial arms." Jama 301, no. 6 (2009): 619-628.

https://doi.org/10.1001/jama.2009.116

2. Oskoei, Mohammadreza Asghari, and Huosheng $\mathrm{Hu}$. "Myoelectric control systems-A survey." Biomedical Signal Processing and Control 2, no. 4 (2007): 275294.

https://doi.org/10.1016/j.bspc.2007.07.009
3. Cortes, Corinna, and Vladimir Vapnik. "Support vector machine [J]." Machine learning 20, no. 3 (1995): 273-297.

https://doi.org/10.1007/BF00994018

4. Dhariwal, S. and Palaniappan, S. (2019) 'An efficient approach for semantic image classification using normalization method', International Journal of Advanced Trends in Computer Science and Engineering, 8(4), pp. 1268-1274. https://doi.org/10.30534/ijatcse/2019/37842019

5. Nasharuddin, N. A., Mohd Yusoff, N. S. and Ali, S. K. (2019) 'Multi-feature vegetable recognition using machine learning approach on leaf images', International Journal of Advanced Trends in Computer Science and Engineering, 8(4), pp. 1789-1794. https://doi.org/10.30534/ijatcse/2019/110842019

6. Waris, Muhammad Asim, Mohsin Jamil, Yasar Ayaz, and Syed Omer Gilani (2014). Classification of Functional Motions of Hand for Upper Limb Prosthesis with Surface Electromyography.International Journal of Biology and Biomedical Engineering 8:15-20.

7. R. N. Khushaba, M. Takruri, S. Kodagoda, and G. Dissanayake, "Toward Improved Control of Prosthetic Fingers Using Surface Electromyogram (EMG) Signals", Expert Systems with Applications, vol 39, no. 12, pp. 10731-10738, 2012.

8. Rafiee, J., M. A. Rafiee, F. Yavari, and M. P. Schoen. "Feature extraction of forearm EMG signals for prosthetics." Expert Systems with Applications 38, no. 4 (2011): 4058-4067.

9. Xie, Renqiang, and Juncheng Cao. "Accelerometerbased hand gesture recognition by neural network and similarity matching." IEEE Sensors Journal 16, no. 11 (2016): 4537-4545.

https://doi.org/10.1109/JSEN.2016.2546942

10. Fariman, H. J., Ahmad, S. A., Marhaban, M. H., Ghasab, M. A., \& Chappell, P. H. (2016). Hand movements classification for myoelectric control system using adaptive resonance theory. Australasian physical \& engineering sciences in medicine, 39(1), 85-102

11. Boostani, Reza, and Mohammad Hassan Moradi. "Evaluation of the forearm EMG signal features for the control of a prosthetic hand." Physiological measurement 24, no. 2 (2003): 309. https://doi.org/10.1088/0967-3334/24/2/307

12. Philipson, L., and R. Sörbye. "Control accuracy and response time in multiple-state myoelectric control of upper-limb prostheses. Initial results in nondisabled volunteers." Medical and Biological Engineering and Computing 25, no. 3 (1987): 289-293.

13. Tkach, Dennis, He Huang, and Todd A. Kuiken. "Study of stability of time-domain features for electromyographic pattern recognition." Journal of neuroengineering and rehabilitation 7, no. 1 (2010): 21.

https://doi.org/10.1186/1743-0003-7-21 
14. Chan ADC, Green GC, "Myoelectric control development toolbox", 30th Conference of the Canadian Medical \& Biological Engineering Society, Toronto, Canada, M0100, 2007.

15. Kim, K. S., Choi, H. H., Moon, C. S., \& Mun, C. W. (2011). Comparison of k-nearest neighbor, quadratic discriminant and linear discriminant analysis in classification of electromyogram signals based on the wrist-motion directions. Current Applied Physics, 11(3), 740-745.

16. Leon, M., J. M. Gutierrez, L. Leija, and R. Munoz. "EMG pattern recognition using Support Vector Machines classifier for myoelectric control purposes." In Health Care Exchanges (PAHCE), 2011 Pan American, pp. 175-178. IEEE, 2011.

17. Zardoshti-Kermani, Mahyar, Bruce C. Wheeler, Kambiz Badie, and Reza M. Hashemi. "EMG feature evaluation for movement control of upper extremity prostheses." IEEE Transactions on Rehabilitation Engineering 3, no. 4 (1995): 324-333.

18. Chen, Xun, and Z. Jane Wang. "Pattern recognition of number gestures based on a wireless surface EMG system." Biomedical Signal Processing and Control 8, no. 2 (2013): 184-192.

19. Hudgins, B., Parker, P., and Scott, R. N. (1993). A new strategy for multifunction myoelectric control. IEEE Trans. Biomed. Eng. 40, 82-94. doi: 10.1109/10.204774

20. Cortes, Corinna, and Vladimir Vapnik. "Support vector machine [J]." Machine learning 20, no. 3 (1995): 273-297.

https://doi.org/10.1007/BF00994018 